Brief article

\title{
Finding words in a language that allows words without vowels
}

\author{
Abder El Aissati a , James M. McQueen ${ }^{\text {b,c,d }}$, Anne Cutler ${ }^{\text {b,d,e,* }}$ \\ ${ }^{\text {a } U n i v e r s i t y ~ o f ~ T i l b u r g, ~ T i l b u r g, ~ T h e ~ N e t h e r l a n d s ~}$ \\ ${ }^{\mathrm{b}}$ Donders Institute for Brain, Cognition and Behaviour, Centre for Cognition, Radboud University Nijmegen, Nijmegen, The Netherlands \\ ${ }^{\mathrm{c}}$ Behavioural Science Institute, Radboud University Nijmegen, Nijmegen, The Netherlands \\ ${ }^{\mathrm{d}}$ Max Planck Institute for Psycholinguistics, Nijmegen, The Netherlands \\ ${ }^{\mathrm{e}}$ MARCS Institute, University of Western Sydney, NSW, Australia
}

\section{A R T I C L E I N F O}

\section{Article history:}

Received 30 December 2011

Revised 18 March 2012

Accepted 21 March 2012

Available online 20 April 2012

\section{Keywords:}

Speech segmentation

Possible Word Constraint

Berber

Vowels

Consonants

\begin{abstract}
A B S T R A C T
Across many languages from unrelated families, spoken-word recognition is subject to a constraint whereby potential word candidates must contain a vowel. This constraint minimizes competition from embedded words (e.g., in English, disfavoring win in twin because $t$ cannot be a word). However, the constraint would be counter-productive in certain languages that allow stand-alone vowelless open-class words. One such language is Berber (where $t$ is indeed a word). Berber listeners here detected words affixed to nonsense contexts with or without vowels. Length effects seen in other languages replicated in Berber, but in contrast to prior findings, word detection was not hindered by vowelless contexts. When words can be vowelless, otherwise universal constraints disfavoring vowelless words do not feature in spoken-word recognition.
\end{abstract}

(c) 2012 Elsevier B.V. All rights reserved.

\section{Introduction}

Vocabularies, though large, are made from just a handful of phonemes, so that inevitably words are at least temporarily ambiguous; word begins with were, phoneme begins like phone and contains own, ambiguous contains am, big, you and us. In print, inter-word spaces define word, phoneme, ambiguous as units. But speech contains no such consistent cues; utterances are fast and continuous. Universally, listeners must work out not only what the constituent words are, but also where they are.

In fact, listeners accomplish the task of segmenting speech into words with relative ease, and this is because their speech processing becomes adapted to language-specific structure. Thus segmentation exploits language rhythm, showing sensitivity to stress in English and Dutch (Cutler \& Norris, 1988; Vroomen, van Zon, \& de Gelder,

\footnotetext{
* Corresponding author. Address: Max Planck Institute for Psycholinguistics, PO Box 310, 6500 AH Nijmegen, The Netherlands. Tel.: +31 24 352 1377; fax: +3124352 1213.

E-mail address: anne.cutler@mpi.nl (A. Cutler).
}

1996), to syllabic patterning in French, Spanish, and Korean (Kim, Davis, \& Cutler, 2008; Mehler, Dommergues, Frauenfelder, \& Segui, 1981; Pallier, Sebastián-Gallés, Felguera, Christophe, \& Mehler, 1993), and to moraic structure in Japanese and Telugu (Murty, Otake, \& Cutler, 2007; Otake, Hatano, Cutler, \& Mehler, 1993). Segmentation also draws on language-specific sequence constraints (Dumay, Frauenfelder, \& Content, 2002; McQueen, 1998; Suomi, McQueen, \& Cutler, 1997; Weber \& Cutler, 2006), and on word-edge probabilities (Banel \& Bacri, 1997; Van der Lugt, 2001). All of these effects are defined across a languagespecific vocabulary, so their use requires experience with the words of a language.

Segmentation also shows patterns that are quite constant across vocabularies. One consistent effect is the Possible Word Constraint (PWC; Norris, McQueen, Cutler, \& Butterfield, 1997). This constrains competing word forms activated by incoming speech, and can be formulated as: "do not accept any available word candidate if accepting it means that a vowelless residue of the input remains".

A speech input can be compatible with many words. The string very few words have no embeddings supports 
not only these six words, but also many embedded forms (you in few, were or whirr or err or erred in word, owe in no, bed and bedding in embedding). The PWC is effective in ruling out such spuriously available candidates - six of the eight above are ruled out, as indeed are $73 \%$ of all embeddings in the English lexicon (Cutler, McQueen, Jansonius, \& Bayerl, 2002).

PWC effects were discovered with word-spotting experiments (see Fig. 1). In word-spotting, listeners hear nonsense sequences (e.g., obzel, crithish, bookving, etc.) and press a button whenever they detect a real word (e.g., book) in any string. English listeners spotted egg more easily in, for instance, maffegg than fegg. Contexts appended to word-spotting targets are never themselves words; however, maff might have been a word (it resembles mat, muff, graph), while $f$ is unlike any English word. The Possible Word Constraint thus rules out residues which could not be words because they do not contain a vowel, while sparing residues which contain a vowel and thus could be as yet unknown words. The phonemic repertoires of all languages contain vowels and consonants, so that this constraint could apply universally.

As Fig. 1 shows, the original English findings replicated in many other languages. Some, with quite different phonological structure, were chosen to test the universality of the PWC. Sesotho, for instance, has no stand-alone monosyllabic words, and Cantonese has a lexicon built from a quite small set of morphemes appearing in very many combinations. In each of these cases, a residue was disfavored only if it was vowelless (Cutler, Demuth, \&
McQueen, 2002; Yip, 2004). It did not matter whether or not the residue could be a stand-alone word in the listener's language; all that mattered was the presence of a vowel. This suggested that the constraint did not depend upon the contents of a given vocabulary, but instead operated in the same way in all languages. Possible words are universal in the sense that across languages it is nearly always the case that syllables, and hence words, must minimally contain a vowel.

Critical for the universality of the PWC, then, are those few languages in which it is not the case that syllables must minimally contain a vowel. These unusual languages, that allow syllables without vowels and vowelless stand-alone words, and in which any consonant can serve as a syllabic nucleus, have exercised phonologists for decades. One of the most well-known such cases is Berber.

In Tarifiyt Berber, for example, the verb work is [xdm]. The phonology of both Tarifiyt and its close dialectal relative Tashelhiyt have been well described (Chami, 1979; Chtatou, 1982; El Aissati, 1989, for Tarifiyt; Boukous, 1987; Dell \& Elmedlaoui, 1985; Ridouane, 2008, for Tashelhiyt; see these references for more examples). In Tarifiyt and Tashelhiyt, syllables need not contain vowels, or even continuant consonants as nuclei. Even voiceless obstruents, the least sonorous of segments, may constitute syllables and serve as syllable nuclei. Ridouane (2008) measured vocal fold vibration and glottal opening in Tashelhiyt speech production; no vowel gestures were observed in syllables transcribed as vowelless.
English
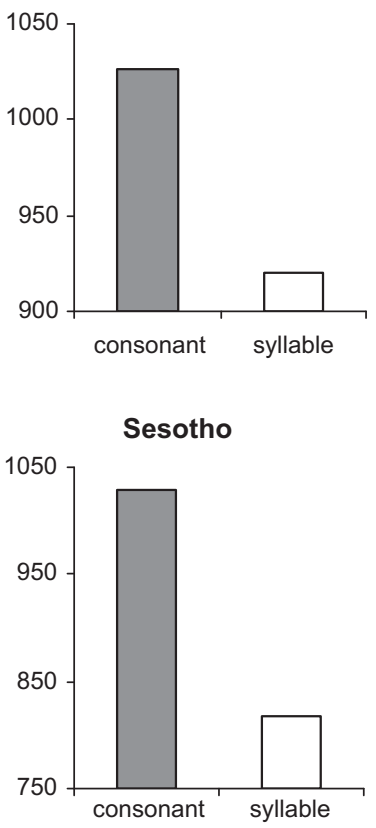

French

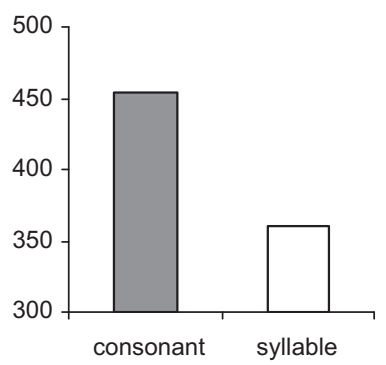

Cantonese

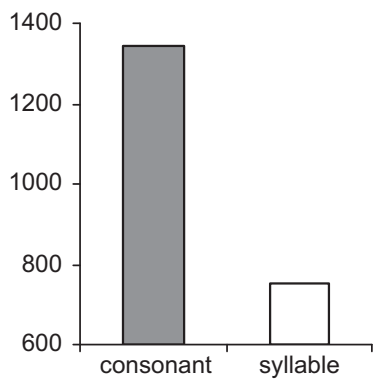

German

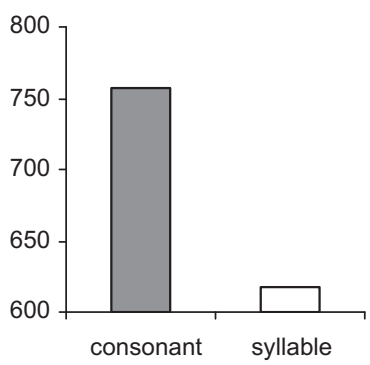

Japanese

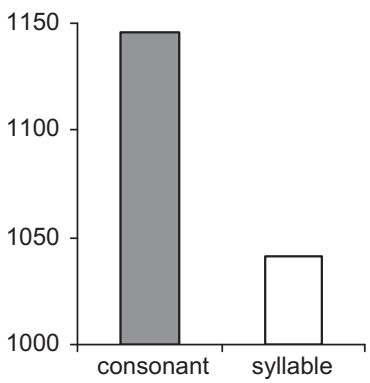

Fig. 1. Word-spotting evidence of the Possible Word Constraint (PWC) in six languages. Mean response times show in each case that detection of a word affixed to a prior context is slower if the context consists only of a consonant than if the context contains a vowel. Thus English egg is detected more slowly in fegg than in maffegg (top left; data from Norris et al., 1997; French data from Dumay et al., 2002; German data from Hanulíková, Mitterer, and McQueen, 2011; Sesotho data from Cutler, Demuth, et al., 2002; Cantonese data from Yip, 2004; Japanese data from McQueen et al., 2001). 
Since the PWC would thus rule out many existing Berber words, it is a real possibility that it will not be applied in Berber. To test this, we examined speech segmentation in Berber with word-spotting. Native Berber speakers listened to nonsense sequences and responded whenever they spotted any real word. The context appended to embedded words was either a consonant or a syllable. The PWC prediction is that word detection will be harder with consonant contexts than with any context containing a vowel. We tested in both the Tarifiyt and Tashelhiyt Berber dialects; the phonological literature on the latter is somewhat more extensive, but the two varieties are structurally quite similar. We here report only the Tarifiyt results, because only for that dialect could we control the competitor population for words within their contexts. Number of competitors becoming available as a function of the context itself, or the word plus its context, influences word-spotting accuracy and speed (Norris, McQueen, \& Cutler, 1995; Van der Lugt, 2001; Vroomen \& de Gelder, 1995). Competitor populations can be checked if adequate dictionary resources are available; for Tarifiyt this was indeed so, but resources of the necessary size and completeness could not be accessed for Tashelhiyt. ${ }^{1}$

\section{Experiment}

\subsection{Participants}

Forty-two student volunteers from Nador, Morocco, all native speakers of Tarifiyt Berber without hearing problems, participated for payment.

\subsection{Materials}

Thirty-nine Tarifiyt words were selected, from one to three syllables in length, all beginning with a ConsonantVowel (CV) sequence. ${ }^{2}$ Nonsense sequences for each target word were made by adding either a single-consonant $(C)$ context or a syllabic context (VC) onto the beginning of each word. Seven different consonants $(/ \mathrm{q} /, / \mathrm{h} /, / 3 /, / \mathrm{z} /, / \mathrm{s} /, / \mathrm{b} /$ and $/ \mathrm{y} /$, transcribed here as $q, h, j, z,<, b$ and gh respectively) were used in these contexts. None of these are stand-alone words or morphemes but all may be syllabic nuclei. The same consonant was used as the single-consonant context and as the consonant in the syllable context of a given word (e.g., for fad, 'thirst', the nonsense sequences were ghfad and aghfad). The sequences of consonants straddling target-word onsets (e.g., ghf for fad) all occur word-internally in Tarifiyt, and thus do not signal phonotactically mandatory boundaries. The number of competitor words beginning with the sequence context + word onset (e.g., for fad: ghfar 'pardon[V]', aghfar 'act of pardoning', ghfel 'trick[V]') was matched as closely as possible across context conditions (mean competitor count beginning context + CV .26 for consonant contexts [ghfar], .26 for syllable contexts [aghfar],

\footnotetext{
1 A full description of the Tashelhiyt materials, experiment and results can be found in Supplementary material.

2 The materials are listed in full in Supplementary material.
}

beginning context $+\mathrm{C} .87$ for consonant contexts [ghfel, ghfar], .46 for syllable contexts [aghfar]). A reduced-syllable preceding context was also recorded for each target word. ${ }^{3}$ An additional 84 fillers, nonsense sequences containing no real words, were made by analogy to the target-bearing items (CV-initial nonwords were preceded by a single consonant or a VC syllable with a full vowel), as were 18 practice items (six nonsense sequences containing words and 12 without). Each item was recorded multiple times.

The stimuli were recorded directly to computer in a sound-attenuated booth by a male native speaker of Tarifiyt who was unaware of the experiment's purpose. Using Praat, the target-bearing items were made by cross-splicing. All cuts were made at zero crossings at the onset of target word initial consonants, as determined auditorily and by visual inspection of waveforms and spectrograms. A clearly-articulated token of each target word was selected and excised from a recording of that word in the reduced-syllable context, then clearly-articulated tokens of each context for each target were selected and excised from recordings with the target, and the contexts and their respective targets were then spliced together. Splices created no noticeable auditory discontinuities. Fillers and practice items were not spliced; a clearly-articulated token of each of these was selected.

\subsection{Procedure}

Different versions of the experiment were constructed, each having the practice block and an experimental block containing all fillers and one version of each target, with context type counterbalanced across versions. Target-bearing and filler items occurred in quasi-random order such that at least one filler always separated any two targetbearers. Running order of targets and fillers was identical in each version.

Participants were randomly assigned to an experimental version and tested individually in a quiet room, with the auditory stimuli presented over headphones. Participants were told that they would hear nonsense sequences and should try to spot any real Tarifiyt words embedded at the end of the sequences. They were asked to press a response button (with a finger of their dominant hand) as quickly as possible if they spotted a real word, and then to say that word aloud. These oral responses were recorded. Stimulus presentation (InterStimulus Interval: $3.5 \mathrm{~s}$.) and logging of button-press latencies were controlled by NESU software on a laptop computer.

Oral responses were examined, and button presses accompanied by no oral response or by a wrong word response ( $<1 \%$ of all experimental trials) were treated as errors. Reaction Times (RTs) were measured from stimulus onset, and adjusted prior to data analysis by subtracting the total duration of the appropriate stimulus from each RT, giving RT from target offset.

\footnotetext{
${ }^{3}$ A reduced-vowel condition was also tested. In the event this condition also did not differ from the two conditions reported here. See Supplementary material for more detail.
} 


\subsection{Results}

Data from one participant who missed two-thirds of all targets was excluded, and data from another was lost due to technical problems. Five targets (mun, niy, lajar, batata, and <am) were missed, overall, by more than $60 \%$ of the participants and responses to these items were also excluded. Mean RTs in each context condition for the remaining participants are shown in Fig. 2. As can immediately be seen, the results do not resemble those in the six panels of Fig. 1.

The RT difference between the context conditions was $10 \mathrm{~ms}$ (SE $29 \mathrm{~ms}$ ), an insignificant difference (F1 and F2 < 1). An analysis of errors showed $27 \%$ error in the consonant condition (SE 2.2\%) and 30\% error in the syllable condition (SE 2.8\%) $(\mathrm{F} 1(1,37)=1.34, p>.2, \mathrm{~F} 2(1,33)=1.39$, $p>.2$ ). Both RT and error differences were in the opposite direction from all previous studies. In short, there was no indication at all that the PWC constrains segmentation of Tarifiyt speech.

An additional analysis across participants included number of target-word syllables as a post hoc factor, since this had previously exercised effects (e.g., Norris et al., 1997). Responses to the monosyllabic targets were compared to those to the bisyllabic targets (the four trisyllabic words were excluded). Bisyllabic words were spotted faster and more accurately than monosyllabic words (mean RT difference $=107 \mathrm{~ms} ; \mathrm{F} 1(1,37)=22.01, p<.001$; mean error difference $=28.4 \% ; \quad F(1,37)=47.7, \quad p<.001)$. This advantage for bisyllabic targets is exactly as previously observed. English listeners were also slower and less accurate, by $365 \mathrm{~ms}$ and 39\% on average, on monosyllabic than on bisyllabic targets in preceding contexts (RTs: $\mathrm{F} 1(1,32)=280.27, \quad p<.001$; errors: $\mathrm{F} 1(1,32)=238.81$, $p<.001$; re-analysis of Norris et al., 1997, Experiment 1;

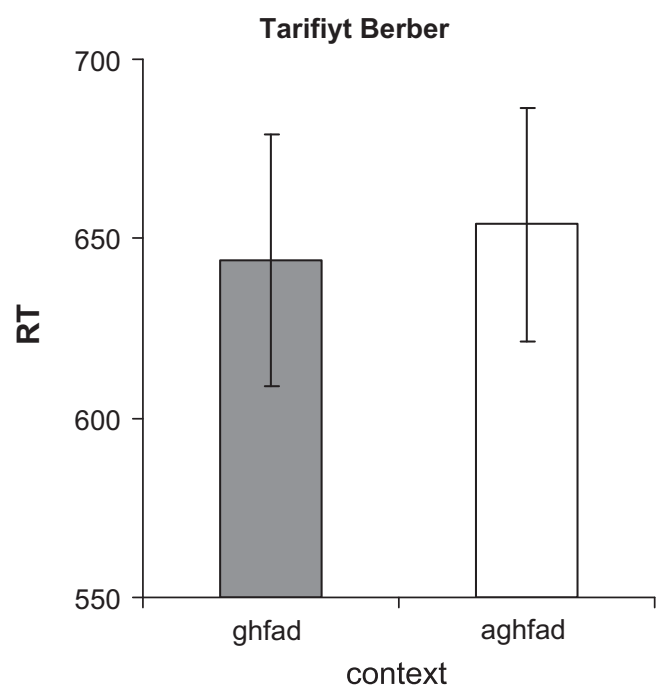

Fig. 2. Word-spotting in Tarifiyt Berber: mean and SE of response time from word offset, as a function of whether the preceding context consisted of a consonant (e.g., fad 'thirst' in ghfad) or a vowel-consonant syllable (e.g., fad in aghfad). re-analysis of Experiment 2 in that study produced fully parallel results). Such parallelism across the studies at least suggests that the Tarifiyt and the English participants performed the word-spotting task in a similar way.

\section{Discussion}

The PWC is not universally applied in segmenting speech. In languages where it could rule out real words, it is not applied. The results of the present study, providing no evidence for the application of the PWC in Berber, contrast dramatically with previous findings.

Words without vowels in their citation form are extremely rare. This fact allows the PWC to benefit processing in most languages. The PWC has been demonstrated with various tasks in English (Newman, Sawusch, \& Wunnenberg, 2011; Norris, McQueen, Cutler, Butterfield, \& Kearns, 2001; Norris et al., 1997), Cantonese (Yip, 2004), Sesotho (Cutler, Demuth, et al., 2002), Dutch (McQueen \& Cutler, 1998; Vroomen \& De Gelder, 1997), German (Hanulíková et al., 2011), French (Dumay et al., 2002; Spinelli, McQueen, \& Cutler, 2003) and Japanese (McQueen, Otake, \& Cutler, 2001). It affects word-form recognition by prelinguistic infants (Johnson, Jusczyk, Cutler, \& Norris, 2003), and a PWC-like lexical viability constraint controls segmentation in British Sign Language (Orfanidou, Adam, Morgan, \& McQueen, 2010).

The present results also contrast interestingly with findings from two other cases of languages where vowelless syllables appear: devoiced vowels in Japanese, and single-consonant prepositions in Slovak. In both cases the PWC proved to hold. In Japanese, vowelless residues inhibited word-spotting even where they could have resulted from application of a devoicing rule (Cutler, Otake, \& McQueen, 2009). In Slovak, vowelless residues that were not prepositions likewise inhibited word-spotting; the four consonants which were actual Slovak prepositions, though, did not exercise inhibition (Hanulíková, McQueen, \& Mitterer, 2010). These four consonants were apparently exempt from the PWC, being in effect honorary vowels, in that they were allowed to be residues which would not inhibit activation of an adjacent word.

Casual speech often produces vowelless sequences, including in all the languages in Fig. 1. But these temporary phenomena do not prevent the PWC from applying in those languages. Nor does a rule, as in Japanese, that causes some sequences to be vowelless. The PWC holds even when a language allows a small set of vowelless words, as in Slovak; it is suspended for the consonants that are these words, but it applies for other consonants. Thus listeners' language experience shapes their listening strategies; most languages do not have vowelless words; but certain languages that do have them require their listeners to adjust listening especially to cope with them. The present results extend relevant coverage to the rare case of languages that allow whole words without vowels, where the PWC is actually unhelpful. Complete Berber multi-word utterances can be vowelless, in fact. Ridouane's (2008) instrumental analyses of Tashelhiyt sentences included strings such as [tsskfftstt tftxtstt] 'you dried it and rolled 
it'. Applying the PWC to utterances like this would make Berber speech comprehension extremely difficult. And indeed, it is not applied.

Together the PWC results contribute significantly to our understanding of universality versus language-specificity in language processing. Universally, vocabularies map meaning to small phonological forms, and utterances consist of continuous sequences of these forms strung together. The need to segment speech in order to understand utterances is universal. Automatic activation of word forms supported by speech signals, and inter-word competition, provide a universally efficient framework for spoken-word recognition. Within that framework, the PWC significantly reduces competition in nearly all languages. Where it works, it works not by applying language-specific rules for stand-alone viability, but simply by reference to the vowel/consonant difference, which is universal (part of all phonologies).

The PWC's insensitivity to language-specific word structure constraints suggests that it is not learned from exposure to words. But it may still be learned, for instance simply from exposure to speech. The acoustic contrast of vowels and consonants, and the relatively greater salience of vowels, induce earlier acquisition of language-specific vowel than consonant representations across languages (Kuhl, Williams, Lacerda, Stevens, \& Lindblom, 1992; Polka \& Werker, 1994), so that the initial core elements of early stored word forms, in any language, are most likely to be vowels.

We cannot yet know whether Berber infants first develop the PWC and then abandon it, or whether they never develop it; no infant listening data from Berber are available. Similarly to many other languages (Snow \& Ferguson, 1977), Berber "baby talk" forms often involve reduplication and have CV syllables, as in sisu for sksu 'couscous' or bubu for aTbub 'sugar lump' (though also, ppspps for uggadn 'urine'; Bynon, 1968; Bynon, 1977). Such input could well encourage Berber-acquiring infants to develop listening strategies that are based on the expected usefulness of the PWC.

Either way, the present results are informative. Berber listeners do not use the PWC, either because their speech input never encouraged its development, or because they tried it out in early vocabulary acquisition, but abandoned it once it proved to rule out candidate words that actually belong in the utterance. Just as Slovak listeners, who otherwise make use of the PWC, have learned to allow four prepositional consonants to count as viable residues, so Berber listeners would in that case have learned from language experience to turn off the PWC entirely.

\section{Acknowledgements}

We thank Mohamed Elmedlaoui, Mohamed Saadouni, Mohammadi Laghzaoui, Abdallah El Mountassir, Maarten Jansonius, Judith Hanssen and Laurence Bruggeman for assistance with materials construction, recording, speech editing, and testing. A partial report of the data was presented at the 12th Conference on Laboratory Phonology, Albuquerque, New Mexico, July 2010. Financial support was provided by the NWO-SPINOZA project "Native and Nonnative Listening".

\section{Appendix A. Supplementary material}

Supplementary data associated with this article can be found, in the online version, at http://dx.doi.org/10.1016/ j.cognition.2012.03.006.

\section{References}

Banel, M.-H., \& Bacri, N. (1997). Reconnaissance de la parole et indices de segmentation metriques et phonotactiques/Cooperation and conflict between metrical cues and phonotactic cues in speech segmentation. L'année Psychologique, 97, 77-112.

Boukous, A. (1987). Phonotactique et domaines prosodiques en berbère/ Phonotactics and prosodic domains in Berber. Thèse de doctorat d'Etat, Université de Paris VIII, Saint-Denis.

Bynon, J. (1968). Berber nursery language. Transactions of the Philological Society, 67, 107-161.

Bynon, J. (1977). The derivational processes relating Berber nursery words to their counterparts in normal inter-adult speech. In C. E. Snow \& C. A. Ferguson (Eds.), Talking to children: Language input and acquisition. Cambridge: Cambridge University Press.

Chami, M. (1979). Un parler amazigh du Rif marocain/A Moroccan Rif Amazigh dialect. Doctorat de troisième cycle, Université de Paris V.

Chtatou, M. (1982). Aspects of the phonology of a Berber dialect of the Rif. Ph.D. dissertation, SOAS, University of London.

Cutler, A., Demuth, K., \& McQueen, J. M. (2002). Universality versus language-specificity in listening to running speech. Psychological Science, 13, 258-262.

Cutler, A., McQueen, J.M., Jansonius, M., \& Bayerl, S. (2002). The lexical statistics of competitor activation in spoken-word recognition. In C. Bow (Ed.), Proceedings of the 9th Australian International Conference on Speech Science and Technology (pp. 40-45). Canberra: Australian Speech Science and Technology Association.

Cutler, A., \& Norris, D. (1988). The role of strong syllables in segmentation for lexical access. Journal of Experimental Psychology: Human Perception and Performance, 14, 113-121.

Cutler, A., Otake, T., \& McQueen, J. M. (2009). Vowel devoicing and the perception of spoken Japanese words. Journal of the Acoustical Society of America, 125, 1693-1703.

Dell, F., \& Elmedlaoui, M. (1985). Syllabic consonants and syllabification in Imdlawn Tashlhiyt Berber. Journal of African Languages and Linguistics, 7, 105-130.

Dumay, N., Frauenfelder, U. H., \& Content, A. (2002). The role of the syllable in lexical segmentation in French: Word-spotting data. Brain and Language, 81, 144-161.

El Aissati, A. (1989). A study of the phonotactics of Asht Touzine Tarifiyt dialect. DES (Diplome des Etudes Superieures) Thesis. Mohamed V University, Rabat.

Hanulíková, A., McQueen, J. M., \& Mitterer, H. (2010). Possible words and fixed stress in the segmentation of Slovak speech. Quarterly Journal of Experimental Psychology, 63, 555-579.

Hanulíková, A., Mitterer, H., \& McQueen, J. M. (2011). Effects of first and second language on segmentation of non-native speech. Bilingualism: Language and Cognition, 14, 506-521.

Johnson, E. K., Jusczyk, P. W., Cutler, A., \& Norris, D. (2003). Lexical viability constraints on speech segmentation by infants. Cognitive Psychology, 46, 65-97.

Kim, J., Davis, C., \& Cutler, A. (2008). Perceptual tests of rhythmic similarity: II. Syllable rhythm. Language and Speech, 51, 342-358.

Kuhl, P., Williams, K. A., Lacerda, F., Stevens, K. N., \& Lindblom, B. (1992). Linguistic experience alters phonetic perception in infants by 6 months of age. Science, 255, 606-608.

McQueen, J. M. (1998). Segmentation of continuous speech using phonotactics. Journal of Memory and Language, 39, 21-46.

McQueen, J.M., \& Cutler, A. (1998). Spotting (different types of) words in (different types of) context. In R.A. Mannell \& J. Robert-Ribes (Eds.), Proceedings of the 5th International Conference on Spoken Language Processing (vol. 6, pp. 2791-2794). Sydney: Australian Speech Science and Technology Association.

McQueen, J. M., Otake, T., \& Cutler, A. (2001). Rhythmic cues and possibleword constraints in Japanese speech segmentation. Journal of Memory and Language, 45, 103-132.

Mehler, J., Dommergues, J.-Y., Frauenfelder, U., \& Segui, J. (1981). The syllable's role in speech segmentation. Journal of Verbal Learning and Verbal Behavior, 20, 298-305.

Murty, L., Otake, T., \& Cutler, A. (2007). Perceptual tests of rhythmic similarity: I. Mora rhythm. Language and Speech, 50, 77-99. 
Newman, R. S., Sawusch, J. R., \& Wunnenberg, T. (2011). Cues and cue interactions in segmenting words in fluent speech. Journal of Memory and Language, 64, 460-476.

Norris, D., McQueen, J. M., \& Cutler, A. (1995). Competition and segmentation in spoken word recognition. Journal of Experimental Psychology: Learning, Memory, and Cognition, 21, 1209-1228.

Norris, D., McQueen, J. M., Cutler, A., \& Butterfield, S. (1997). The possibleword constraint in the segmentation of continuous speech. Cognitive Psychology, 34, 191-243.

Norris, D., McQueen, J. M., Cutler, A., Butterfield, S., \& Kearns, R. (2001). Language-universal constraints on speech segmentation. Language and Cognitive Processes, 16, 637-660.

Orfanidou, E., Adam, R., Morgan, G., \& McQueen, J. M. (2010). Recognition of signed and spoken language: Different sensory inputs, the same segmentation procedure. Journal of Memory and Language, 62, $272-283$.

Otake, T., Hatano, G., Cutler, A., \& Mehler, J. (1993). Mora or syllable? Speech segmentation in Japanese. Journal of Memory and Language, 32, 358-378.

Pallier, C., Sebastián-Gallés, N., Felguera, T., Christophe, A., \& Mehler, J. (1993). Attentional allocation within the syllabic structure of spoken words. Journal of Memory and Language, 32, 373-389.

Polka, L., \& Werker, J. F. (1994). Developmental changes in perception of nonnative vowel contrasts. Journal of Experimental Psychology: Human Perception and Performance, 20, 421-435.
Ridouane, R. (2008). Syllables without vowels: Phonetic and phonological evidence from Tashlhiyt Berber. Phonology, 25, 321-359.

Snow, C. E., \& Ferguson, C. A. (Eds.). (1977). Talking to children: Language input and acquisition. Cambridge: Cambridge University Press.

Spinelli, E., McQueen, J. M., \& Cutler, A. (2003). Processing resyllabified words in French. Journal of Memory and Language, 48, 233-254.

Suomi, K., McQueen, J. M., \& Cutler, A. (1997). Vowel harmony and speech segmentation in Finnish. Journal of Memory and Language, 36, 422-444.

van der Lugt, A. H. (2001). The use of sequential probabilities in the segmentation of speech. Perception and Psychophysics, 63, 811-823.

Vroomen, J., \& de Gelder, B. (1995). Metrical segmentation and lexical inhibition in spoken word recognition. Journal of Experimental Psychology: Human Perception and Performance, 21, 98-108.

Vroomen, J., \& de Gelder, B. (1997). Activation of embedded words in spoken word recognition. Journal of Experimental Psychology: Human Perception and Performance, 23, 710-720.

Vroomen, J., van Zon, M., \& de Gelder, B. (1996). Cues to speech segmentation: Evidence from juncture misperceptions and word spotting. Memory and Cognition, 24, 744-755.

Weber, A., \& Cutler, A. (2006). First-language phonotactics in secondlanguage listening. Journal of the Acoustical Society of America, 119, 597-607.

Yip, M. C. (2004). Possible-word constraints in Cantonese speech segmentation. Journal of Psycholinguistic Research, 33, 165-173. 